PROCEEDINGS OF THE

AMERICAN MATHEMATICAL SOCIETY

Volume 136, Number 6, June 2008, Pages 1987-1995

S 0002-9939(08)09323-4

Article electronically published on February 15, 2008

\title{
A NEW APPROACH TO RELATIVELY NONEXPANSIVE MAPPINGS
}

\author{
RAFA ESPÍNOLA
}

(Communicated by Jonathan M. Borwein)

\begin{abstract}
In this paper we study the nonexpansivity of the so-called relatively nonexpansive mappings. A relatively nonexpansive mapping with respect to a pair of subsets $(A, B)$ of a Banach space $X$ is a mapping defined from $A \cup B$ into $X$ such that $\|T x-T y\| \leq\|x-y\|$ for $x \in A$ and $y \in B$. These mappings were recently considered in a paper by Eldred et al. (Proximinal normal structure and relatively nonexpansive mappings, Studia Math. 171 (3) (2005), 283-293) to obtain a generalization of Kirk's Fixed Point Theorem. In this work we show that, for certain proximinal pairs $(A, B)$, there exists a natural semimetric for which any relatively nonexpansive mapping with respect to $(A, B)$ is nonexpansive. This fact will be used to improve one of the two main results from the aforementioned paper by Eldred et al. At that time we will also obtain several consequences regarding the strong continuity properties of relatively nonexpansive mappings and the relation between the two main results from the same work.
\end{abstract}

\section{INTRODUCTION AND PRELIMINARIES}

In a recent paper by Eldred et al. 33 two results about the existence of a fixed point for relatively nonexpansive mappings were obtained. As the authors explain in the introduction, the significance of these two results lies in the fact that a relatively nonexpansive assumption is much weaker than the assumption of nonexpansivity. Our main goal in this work is to take up the problem of the continuity properties of relatively nonexpansive mappings and, in particular, their actual nonexpansive properties. As a consequence of our work we show that, under suitable conditions, these mappings are nonexpansive if we look at them from the right topology. This topology will be given by a semimetric under which any relatively nonexpansive mapping defined on $A \cup B$ and such that $T(A) \subseteq A$ and $T(B) \subseteq B$ is nonexpansive (see below for definitions). This fact will have several consequences, including an improvement of one of the two main results from [3] (Theorem 2.2) where the condition of strict convexity on the space $X$ is replaced by a weaker assumption directly imposed on the proximinal pair. We also prove that relatively nonexpansive mappings are uniformly continuous when $X$ is a uniformly convex space and nonexpansive for $X$ Hilbert or $A$ and $B$ segments. In [3] the authors also present a Ky Fan-like result (Theorem 2.1) for $T$ relatively nonexpansive and

Received by the editors July 18, 2006.

2000 Mathematics Subject Classification. Primary 47H10.

The author was partially supported by the Ministry of Science and Technology of Spain, Grant MTM 2006-13997-CO2-01 and La Junta de Antalucía project FQM-127.

(C)2008 American Mathematical Society
Reverts to public domain 28 years from publication 1987 
such that $T(A) \subseteq B$ and $T(B) \subseteq A$. We close this work by giving an application of our main result to this situation and, although this time our result is not as strong as the one given in [3], we show that, under more general conditions than those of Theorem 2.2 in [3], Theorem 2.2 is actually a consequence of Theorem 2.1.

Let $X$ be a Banach space and $A$ and $B$ two nonempty subsets of $X$. We will use the following notation:

$$
\begin{gathered}
\delta(A, B)=\sup \{\|x-y\|: x \in A, y \in B\} \\
\delta(x, B)=\sup \{\|x-y\|: y \in A\} \\
\operatorname{dist}(A, B)=\inf \{\|x-y\|: x \in A, y \in B\} .
\end{gathered}
$$

A relatively nonexpansive mapping between two subsets of a Banach space is defined as follows.

Definition 1.1. Let $A$ and $B$ be two nonempty subsets of a Banach space. Then the mapping $T: A \cup B \rightarrow A \cup B$ is a relatively nonexpansive mapping between $A$ and $B$ if $\|T x-T y\| \leq\|x-y\|$ for any $x \in A$ and $y \in B$.

In this work we will need to distinguish among different kinds of proximinal pairs of sets. This is given by the following definitions.

Definition 1.2. A pair $(A, B)$ of subsets of a metric space is said to be a proximinal pair if for each $(x, y) \in A \times B$ there exists $\left(x^{\prime}, y^{\prime}\right) \in A \times B$ such that

$$
\left\|x-y^{\prime}\right\|=\left\|x^{\prime}-y\right\|=\operatorname{dist}(A, B) .
$$

If, additionally, we impose the condition that the pair of points $\left(x^{\prime}, y^{\prime}\right) \in A \times B$ is unique for each $(x, y) \in(A, B)$, then we say that the pair $(A, B)$ is a sharp proximinal pair.

Definition 1.3. Let $A$ and $B$ be two nonempty subsets of a Banach space $X$. We will say that $A$ and $B$ are proximinal parallel sets if the following two conditions are fulfilled:

(1) $(A, B)$ is a sharp proximinal pair.

(2) $B=A+h$ for a certain $h \in X$.

Remark 1.4. Notice that, in this case, $a+h$ is the proximinal point in $B$ to $a$ for any $a \in A$.

We will say that a pair of sets $(A, B)$ in a Banach space satisfies a property if each of the sets $A$ and $B$ has that property. Thus $(A, B)$ is said convex if both $A$ and $B$ are convex; $(A, B) \subseteq(C, D) \Leftrightarrow A \subseteq C$ and $B \subseteq D$. The next definition was introduced in [3].

Definition 1.5. A convex pair $\left(K_{1}, K_{2}\right)$ (i.e., both $K_{1}$ and $K_{2}$ are convex sets) in a Banach space is said to have proximinal normal structure if for any closed, bounded, convex proximinal pair $\left(H_{1}, H_{2}\right) \subseteq\left(K_{1}, K_{2}\right)$ for which $\operatorname{dist}\left(H_{1}, H_{2}\right)=\operatorname{dist}\left(K_{1}, K_{2}\right)$ and $\delta\left(H_{1}, H_{2}\right)>\operatorname{dist}\left(H_{1}, H_{2}\right)$, there exists $\left(x_{1}, x_{2}\right) \in H_{1} \times H_{2}$ such that

$$
\delta\left(x_{1}, H_{2}\right)<\delta\left(H_{1}, H_{2}\right), \quad \delta\left(x_{2}, H_{1}\right)<\delta\left(H_{1}, H_{2}\right) .
$$

In 3] the reader can find different conditions which guarantee proximinal normal structure for a given pair of sets.

To deduce our main fixed point result we will need a version of Kirk's Theorem for semimetric spaces. 
Definition 1.6. Let $M$ be a set; then a function $d: M \times M \rightarrow[0, \infty)$ is a semimetric on $M$ if

(1) $d(x, y)=0$ if, and only if, $x=y$.

(2) $d(x, y)=d(y, x)$ for any $x, y \in M$.

Given a semimetric $d$, a $B$-set will be a set like

$$
B(x, r)=\{y \in M: d(x, y) \leq r\} .
$$

Consider the family of admissible subsets $\mathcal{A}(M)$ of $M$ as those nonempty subsets of $M$ which are an intersection of $B$-sets. As usual we will say that $\mathcal{A}(M)$ is compact if any decreasing intersection of elements of $\mathcal{A}(M)$ is nonempty. For $D \subseteq M$, the diameter and radius of $D$ are defined in the usual way as

$$
\operatorname{diam}(D)=\sup \{d(x, y): x, y \in D\}
$$

and

$$
r(D)=\inf _{x \in D} \sup _{y \in D} d(x, y) .
$$

The notion of normal structure was introduced by Brodskii and Milman in [2]. This notion plays a central role in metric fixed point theory due mainly to Kirk's Theorem (see [5]). For more on this concept, metric fixed point theory and related topics, the reader may check the references [1, 4, 7].

Definition 1.7. We say that $\mathcal{A}(M)$ has normal structure if, for $D \in \mathcal{A}(M)$ bounded (with respect to $d$ ) and nonempty, $\operatorname{diam}(D)=r(D)$ implies that $D$ is a singleton.

Nonexpansiveness with respect to a semimetric is defined in the natural way.

Definition 1.8. A mapping $T: M \rightarrow M$ is said to be nonexpansive with respect to the semimetric $d$ if $d(T x, T y) \leq d(x, y)$ for any $x, y \in M$.

An abstract version of Kirk's Fixed Point Theorem is stated in [6]. The following theorem is a particular case for semimetrics of this abstract version.

Theorem 1.9. Let $(M, d)$ be a bounded semimetric space such that $\mathcal{A}(M)$ is compact and has normal structure. Then every nonexpansive mapping $T: M \rightarrow M$ has a fixed point.

For the sake of completeness we state the next two theorems which are, respectively, Theorems 2.1 and 2.2 in [3].

Theorem 1.10. Let $(A, B)$ be a nonempty, weakly compact convex pair in a Banach space, and suppose $(A, B)$ has proximinal normal structure. Let $T: A \cup B \rightarrow A \cup B$ be a relatively nonexpansive mapping (between $A$ and $B$ ) such that $T(A) \subseteq B$ and $T(B) \subseteq A$. Then there exists $(x, y) \in A \times B$ such that $\|x-T x\|=\|y-T y\|=$ $\operatorname{dist}(A, B)$.

Theorem 1.11. Let $(A, B)$ be a nonempty, weakly compact convex pair in a strictly convex Banach space, and suppose $(A, B)$ has proximinal normal structure. Let $T: A \cup B \rightarrow A \cup B$ be a relatively nonexpansive mapping (between $A$ and $B$ ) such that $T(A) \subseteq A$ and $T(B) \subseteq B$. Then there exist $x_{0} \in A$ and $y_{0} \in B$ such that $T x_{0}=x_{0}, T y_{0}=y_{0}$ and $\left\|x_{0}-y_{0}\right\|=\operatorname{dist}(A, B)$. 


\section{MAIN RESULTS}

Let $A$ and $B$ be proximinal parallel sets, so we can write $B=A+h$ as in Definition 1.3. Also denote by $a^{\prime}=a+h$ for $a \in A$ and $b^{\prime}=b-h$ for $b \in B$ the proximinal points from $B$ and $A$, respectively, to $a$ and $b$. Let us further consider the set $C=A+2 h$. It is immediate to see that $A, B$ and $C$ are pairwise proximinal parallel sets. We begin with the following technical result:

Proposition 2.1. Let $A$ and $B$ be proximinal parallel sets and $T$ a relatively nonexpansive mapping defined on $A \cup B$ such that $T(A) \subseteq A$ and $T(B) \subseteq B$. Then $T(a+h)=T a^{\prime}=T a+h$. Moreover there is a unique extension (which is obtained by translation) of $T$ to $C$ such that $T(C) \subseteq C$ and $T$ is relatively nonexpansive on $B \cup C$.

Proof. We first show that $T a^{\prime}=T a+h$ for any $a \in A$. This follows from the fact that $\left\|T a-T a^{\prime}\right\| \leq\left\|a-a^{\prime}\right\|=\operatorname{dist}(A, B)$; therefore $T a^{\prime}=(T a)^{\prime}$ and so the claim holds. Now, given $x \in C$ then $x=a+2 h$ for a certain $a \in A$; therefore we can make $x=a^{\prime \prime}$. Define

$$
T\left(a^{\prime \prime}\right)=(T a)^{\prime \prime} .
$$

Then, for $b^{\prime} \in B$ and $c^{\prime \prime} \in C$ we have

$$
\begin{gathered}
\left\|T b^{\prime}-T c^{\prime \prime}\right\|=\left\|T b+h-\left(T c^{\prime}+h\right)\right\| \\
=\left\|T b-T c^{\prime}\right\| \leq\left\|b-c^{\prime}\right\|=\left\|b+h-\left(c^{\prime}+h\right)\right\|=\left\|b^{\prime}-c^{\prime \prime}\right\| .
\end{gathered}
$$

Consider the sets $A, B$ and $C$ as above with $d=\operatorname{dist}(A, B)$. In the following lemma we define a semimetric which is going to play a main role in this work.

Lemma 2.2. Let $d_{1}: B \times B \rightarrow[0, \infty)$ be defined as

$$
d_{1}(x, y)=\inf \{r>0: y \in B(x-h, d+r) \cap B(x+h, d+r)\} .
$$

Then $d_{1}$ defines a semimetric on $B$.

Proof. The property that $d_{1}(x, y)=0 \Leftrightarrow x=y$ is an immediate consequence of the fact that $(A, B)$ is a sharp proximinal pair. For symmetry, let $r>d_{1}(x, y)$; then

$$
\|x-(y-h)\|=\|x-y+h\|=\|y-(x+h)\| \leq d+r
$$

and

$$
\|x-(y+h)\|=\|x-y-h\|=\|y-(x-h)\| \leq d+r
$$

hence

$$
x \in B(y-h, d+r) \cap B(y+h, d+r),
$$

and the proof is completed.

The $B$-sets of $d_{1}$ will be denoted as

$$
B_{1}(x, r)=\left\{y \in B: d_{1}(x, y) \leq r\right\},
$$

in contrast to $B(x, r)$, which stands for the usual closed ball of center $x$ and radius $r$ defined by the norm of $X$. Notice that the $B$-sets defined by $d_{1}$ are intersections of norm balls and so they are closed and convex in the usual sense.

The next theorem establishes the relation between relatively nonexpansive mappings with respect to proximinal parallel sets and the semimetric defined by these parallel sets. 
Theorem 2.3. Let $A$ and $B$ be proximinal parallel sets and $T: A \cup B \rightarrow A \cup B$ a relatively nonexpansive mapping such that $T(A) \subseteq A$ and $T(B) \subseteq B$. Then, for any $x, y \in B$,

$$
T y \in B_{1}\left(T x, d_{1}(x, y)\right) \text {, }
$$

i.e., $d_{1}(T x, T y) \leq d_{1}(x, y)$, and so $T$ is $d_{1}$-nonexpansive.

Proof. Let $r=d_{1}(x, y)$ and consider $B_{1}(x, r)$. It is enough if we show that $T\left(B_{1}(x, r)\right) \subseteq B_{1}(T x, r)$. Let $y \in B_{1}(x, r)$; then

$$
\max \{\|y-(x-h)\|,\|y-(x+h)\|\} \leq d+r .
$$

But $T$ is relatively nonexpansive between $A$ and $B$, and $B$ and $C$, so

$$
\|T y-T(x-h)\| \leq\|y-(x-h)\| \leq d+r
$$

and

$$
\|T y-T(x+h)\| \leq\|y-(x+h)\| \leq d+r .
$$

Moreover, $T(x-h)=T(x)-h$ and $T(x+h)=T(x)+h$. Therefore, by definition, $T y \in B_{1}(T x, r)$.

The following theorem is an immediate consequence of Theorems 1.9 and 2.3 .

Theorem 2.4. Let $A$ and $B$ be proximinal parallel bounded sets and $\left(B, d_{1}\right)$ the semimetric space given by Lemma 2.2. If $\mathcal{A}(B)$ is compact and has normal structure, and $T: A \cup B \rightarrow A \cup B$ is a relatively nonexpansive mapping with $T(A) \subseteq A$ and $T(B) \subseteq B$, then there exists $b \in B$ such that $T(b)=b$. Consequently $b-h \in A$ is another fixed point of $T$.

\section{Consequences}

The first goal in this section is to study the connection between Theorem 1.11 and Theorem 2.4. We begin with a result for strictly convex Banach spaces (for details on strictly convex spaces the reader may check the references [1, 4, 7]).

Lemma 3.1. Let $X$ be a strictly convex Banach space. If $(A, B)$ is a proximinal pair in $X$, then $A$ and $B$ are proximinal parallel sets.

Proof. The fact that $(A, B)$ is a sharp proximinal pair immediately follows from the strict convexity of $X$. Now let $x$ and $y$ be in $A$, and $x^{\prime}, y^{\prime}$ their proximinal points in $B$. Let $\vec{u}=y-x$ and $\vec{v}=y^{\prime}-x^{\prime}$ and make $x_{\lambda}=x+\lambda \vec{u}$ and $x_{\lambda}^{\prime}=x^{\prime}+\lambda \vec{v}$. Since for $\lambda=0$ and $\lambda=1$ we have $d_{\lambda}=\left\|x_{\lambda}-x_{\lambda}^{\prime}\right\|=d=\operatorname{dist}(A, B)$, the convexity of the unit ball and the fact that $(A, B)$ is a proximinal pair imply that $d_{\lambda}=d$ for $\lambda \in(0,1)$. If it is the case that $\vec{u} \neq \vec{v}$, then it is easy to see that there exists a segment of positive length in the unit sphere of $X$, which contradicts the strict convexity of $X$. Therefore $\vec{u}=\vec{v}$. Fix $b$ any point in $A$ and make $h=b-b^{\prime}$; then the proof is completed since for any $a \in A$ it happens that $a^{\prime}=a+h$.

One may wonder if the above remains true for any sharp proximinal pair. The next example shows that this is not the case.

Example 3.2. Let $X$ be $\mathbb{R}^{3}$ with the $\ell_{1}$ norm and take $A$ as the segment joining $(0,0,0)$ with $(0,1,0)$ and $B$ as the segment joining $(0,0,1)$ with $(1,1,0)$. Then it is not hard to see that $(A, B)$ is a sharp proximinal pair but they are not proximinal parallel sets. Notice also that $A$ and $B$ do not even have the same length! 
The following result states the relation between the proximinal normal structure of convex pairs given by Definition 1.5 and normal structure with respect to $d_{1}$ given by Definition 1.7 replacing admissible subsets with convex ones.

Theorem 3.3. Let $X$ be a strictly convex Banach space and $(A, B)$ a proximinal pair. Then $(A, B)$ has proximinal normal structure if and only if the family of convex subsets of $B$ has normal structure with respect to $d_{1}$.

Proof. $\Rightarrow$ : Let $(A, B)$ have proximinal normal structure and let $D \subseteq B$ be nonempty closed bounded convex and such that $\operatorname{diam}_{1}(D)>0$. Then the pair $(D-h, D)$ is a closed bounded convex proximinal sub-pair of $(A, B)$ as in Definition 1.5. The strict convexity of the space implies that $\operatorname{dist}(D-h, D)<\delta(D-h, D)$; hence there exists $x_{1} \in D-h$ and $x_{2} \in D$ such that

$$
\delta\left(x_{1}, D\right)<\delta(D-h, D), \quad \delta\left(x_{2}, D-h\right)<\delta(D-h, D) .
$$

Then $x_{2}+h \in D+h$ and, by translation, $\delta\left(x_{2}+h, D\right)<\delta(D, D+h)$. Make $r_{1}=\delta\left(x_{1}, D\right), r_{2}=\delta\left(x_{2}+h, D\right), a=x_{1}+h$ and $b=x_{2}$. Then, for $z \in D$,

$$
\begin{aligned}
& \left\|\frac{a+b}{2}+h-z\right\| \leq\left\|\frac{a+h-z}{2}\right\|+\left\|\frac{b+h-z}{2}\right\| \\
& \leq \frac{\mathrm{d}_{1}(a, z)}{2}+\frac{r_{2}}{2} \leq \frac{\operatorname{diam}_{1}(D)}{2}+\frac{r_{2}}{2}<\operatorname{diam}_{1}(D)
\end{aligned}
$$

and, similarly,

$$
\begin{aligned}
\| \frac{a+b}{2} & -h-z\|\leq\| \frac{a-h-z}{2}\|+\| \frac{b-h-z}{2} \| \\
& \leq \frac{r_{1}}{2}+\frac{\operatorname{diam}_{1}(D)}{2}<\operatorname{diam}_{1}(D) .
\end{aligned}
$$

So $r_{1}(D) \leq \delta_{1}\left(\frac{a+b}{2}, D\right) \leq \max \left\{\frac{\operatorname{diam}_{1}(D)}{2}+\frac{r_{2}}{2}, \frac{r_{1}}{2}+\frac{\operatorname{diam}_{1}(D)}{2}\right\}<\operatorname{diam}_{1}(D)$. Therefore $B$ has normal structure with respect to its convex subsets and, in particular, $\mathcal{A}(B)$ has normal structure as in Definition 1.7.

$\Leftarrow$ : Let $\left(H_{1}, H_{2}\right)$ be a closed bounded and convex proximinal sub-pair of $(A, B)$ such that

$$
\operatorname{dist}(A, B)=\operatorname{dist}\left(\mathrm{H}_{1}, \mathrm{H}_{2}\right) \text { and } \delta\left(H_{1}, H_{2}\right)>\operatorname{dist}\left(H_{1}, H_{2}\right) .
$$

It must be the case that $\left(H_{1}, H_{2}\right)=(D-h, D)$ with $D=H_{2}$. The $d_{1}$-normal structure of $B$ implies that there exists $a \in D$ such that $\delta_{1}(a, D)<\operatorname{diam}_{1}(D)$. Now it suffices to take $x_{1}=a-h \in D-h$ and $x_{2}=a \in D$ to complete the proof.

As a corollary we obtain Theorem 1.11.

Corollary 3.4 (Theorem 1.11). Let $(A, B)$ be a nonempty, weakly compact convex pair in a strictly convex Banach space, and suppose that $(A, B)$ has proximinal normal structure. Suppose that $T: A \cup B \rightarrow A \cup B$ is relatively nonexpansive with $T(A) \subseteq A$ and $T(B) \subseteq B$; then there exists $x_{0} \in A$ and $y_{0} \in B$ such that

$$
T x_{0}=x_{0}, T y_{0}=y_{0} \text {, and }\left\|x_{0}-y_{0}\right\|=\operatorname{dist}(A, B) \text {. }
$$

Proof. As it is shown in [3, there exists $\left(A_{0}, B_{0}\right) \subseteq(A, B)$ a pair of proximinal parallel sets such that $T$ is relatively nonexpansive with respect to them and $T\left(A_{0}\right) \subseteq A_{0}$ and $T\left(B_{0}\right) \subseteq B_{0}$. Assumptions from the corollary imply that $\left(\mathcal{A}\left(B_{0}\right), d_{1}\right)$ enjoys all the required conditions to apply Theorem 2.4. Hence $T$ has 
a fixed point in $B$. If $y_{0}$ stands for this fixed point, taking $x_{0}=y_{0}-h$ completes the proof.

Next we study the relation between the semimetric $d_{1}$ and the norm of the space $X$. We begin with a rather general fact. Let $(A, B)$ be a proximinal pair in a strictly convex space $X$. Assume that $0 \in \operatorname{relint}(B)$, where $\operatorname{relint}(B)$ stands for the relative interior of $B$, and consider $s p(B)$ to be the linear space generated by $B$. Make $s p(A)=s p(B)-h$ and $s p(C)=s p(B)+h$ for $A, B$ and $C$ as usual.

Proposition 3.5. The sets $s p(A), s p(B)$ and $s p(C)$ are pairwise proximinal parallel sets.

Proof. Let us assume that there exist $a \in \operatorname{sp}(A)$ and $b \in \operatorname{sp}(B)$ such that $\|a-b\| \leq$ $d=\operatorname{dist}(A, B)$ and $b \neq a+h$. Obviously $a \in \operatorname{relint}(a+h+A)$ and $a+h \bar{\epsilon}$ $\operatorname{relint}(B+a+h)$. Therefore, if $[b, a+h]$ stands for the segment joining $b$ with $a+h$,

$$
I=[b, a+h] \cap \operatorname{relint}(B+a+h)
$$

is a positive length segment such that $\|x-a\| \leq d$ for any $x \in I$, which contradicts the fact that $A$ and $B$ are proximinal parallel sets. The same reasoning applied to each case completes the proof.

Consider the following family of sets:

$$
\mathcal{F}=\left\{B_{1}(0, r)=B(-h, d+r) \cap B(h, d+r) \cap s p(B): r \in[0,+\infty)\right\} .
$$

Proposition 3.6. The following properties are true:

i) The $B$-sets of $d_{1}$ on $s p(B)$ are the elements of $\mathcal{F}$ and they are translated within $\operatorname{sp}(B)$.

ii) The relation $d_{1}(x, y) \leq\|x-y\|$ holds for any $x, y \in s p(B)$.

Proof. i) is immediate. For ii) it suffices to recall that, as an easy application of the triangle inequality, we have $B(0, r) \subseteq B_{1}(0, r)$ for any $r$.

Our next result applies to uniformly convex Banach spaces (the reader may check references [1, 4, 7, for details on these spaces).

Lemma 3.7. Let $X$ be a uniformly convex Banach space and $A$ and $B$ proximinal parallel subsets of $X$. Then for every $B(x, r)$ in $s p(B)$ there exists $f(r)>0$ such that $B_{1}(x, f(r)) \subseteq B(x, r)$.

Proof. We first prove that there exists a positive constant $k$ such that $B(0,1) \supset$ $B_{1}(0, k)$. If this is not true, by convexity, for each positive $k$ there exists $x_{k} \in \operatorname{sp}(B)$ such that $\left\|x_{k}\right\|=1$ and $x_{k} \in B_{1}(0, k)$. Since $B_{1}(0, k)$ is symmetric with respect to the origin, $-x_{k}$ satisfies the same conditions as $x_{k}$. Hence the segment $\left[x_{k},-x_{k}\right]$ has length 2 and satisfies that

$$
\left[x_{k},-x_{k}\right] \subseteq B(h, d+k) \backslash \operatorname{int}(B(h, d))
$$

for any $k$, which contradicts the uniform convexity of the space. Therefore the claim follows. For a general $r$ we follow similar reasoning. For a general center $x$ we just have to apply the adequate translation.

Next we show that a relatively nonexpansive mapping has good strong continuity properties under suitable conditions. 
Theorem 3.8. Let $(A, B)$ be a proximity pair in a Banach space $X$. Let $T$ : $A \cup B \rightarrow A \cup B$ be a relatively nonexpansive mapping with $T(A) \subseteq A$ and $T(B) \subseteq B$. Then the following statements are true:

i) If $X$ is uniformly convex, then $T$ is a uniformly continuous.

ii) If $X$ is a Hilbert space or $\operatorname{sp}(B)$ is a one dimensional space, then $T$ is nonexpansive.

Proof. i) It immediately follows from Theorem 2.3, ii) of Proposition 3.6 and Lemma 3.7.

ii) The case when $X$ is a Hilbert space is Proposition 3.2 in [3] however it can also be deduced from the fact that

$$
B_{1}(0, r)=B(0, f(r)) \cap s p(B)
$$

for a certain function $f$. This fact is an easy consequence of the Pythagorean Theorem. The situation is similar for $s p(B)$ as a one dimensional space. Since $B_{1}(0, r)$ are symmetric with respect to 0 and convex, they are intervals centered at 0 . Then the conclusion follows in an easy way.

Remark 3.9. Notice that, as it can be easily deduced from the remark on page 288 in 3, a relatively nonexpansive mapping need not be continuous in general.

We finish this work by showing that Theorem 1.10 is actually a consequence of Theorem 1.11 when the pair of sets $(A, B)$ is a pair of proximinal parallel sets. Let now $T: A \cup B \rightarrow A \cup B$ be a relatively nonexpansive mapping such that $T(A) \subseteq B$ and $T(B) \subseteq A$. Then it is easy to see that for $A$ and $B$ proximinal parallel sets, $a \in A$ and $b \in B$,

$$
T(a)-h=T(a+h) \text { and } T(b)+h=T(b-h) .
$$

Define

$$
T^{\prime}(b)=T(b)+h
$$

for $b \in B$.

Lemma 3.10. Let $T$ and $T^{\prime}$ be as above; then $T^{\prime}$ is nonexpansive with respect to $d_{1}$.

Proof. Let $x, y \in B$ with $d_{1}(x, y)=r$; we want to prove that $d_{1}\left(T^{\prime} x, T^{\prime} y\right) \leq r$. Indeed,

$$
T^{\prime} y \in B_{1}\left(T^{\prime} x, r\right) \Leftrightarrow T^{\prime} y \in B\left(T^{\prime} x-h, d+r\right) \cap B\left(T^{\prime} x+h, d+r\right) .
$$

Now,

$$
\begin{gathered}
T^{\prime} y \in B\left(T^{\prime} x-h, d+r\right) \Leftrightarrow\left\|T^{\prime} y-\left(T^{\prime} x-h\right)\right\| \leq d+r \\
\Leftrightarrow\|T y+h-(T x+h-h)\| \leq d+r \Leftrightarrow\|T y-(T x-h)\| \leq d+r \\
\Leftrightarrow\|T x-(T y+h)\| \leq d+r \Leftrightarrow\|T(x)-T(y-h)\| \leq d+r .
\end{gathered}
$$

But, by the relatively nonexpansivity of $T$,

$$
\|T(x)-T(y-h)\| \leq\|x-(y-h)\|
$$

and, by hypothesis,

$$
\|x-(y-h)\| \leq d+r .
$$

We proceed in a similar way to show that $T^{\prime} y \in B\left(T^{\prime} x+h, d+r\right)$, which completes the proof. 
The following theorem is a particular case of Theorem 1.10 .

Theorem 3.11. If $T$ is as above and we are under the conditions of Theorem 2.4, then there exist $a \in A$ and $b \in B$ such that $\|a-T a\|=\|b-T b\|=\operatorname{dist}(A, B)$.

Proof. This theorem follows by applying Theorem 2.4 to the mapping $T^{\prime}$.

The next corollary is a particular case of the above.

Corollary 3.12. If $X$ is strictly convex, then Theorem 1.10 is a consequence of Theorem 1.11

\section{ACKNOWLEDGMENT}

The author would like to thank Andrzej Wiśnicki, from UMCS of Lublin (Poland), for fruitful conversations regarding the subject of this paper.

\section{REFERENCES}

[1] J. M. Ayerbe, T. Domínguez and G. López, Measures of Noncompactness on Metric Fixed Point Theory, Birkhäuser, 1997.

[2] M. S. Brodskii and D. P. Milman, On the center of a convex set (Russian), Dokl. Akad. Nauk SSSR 59 (1948), 837-840. MR0024073 (9:448f)

[3] A. A. Eldred, W. A. Kirk and P. Veeramani, Proximinal normal structure and relatively nonexpansive mappings, Studia Math. 171 (3) (2005), 283-293. MR2188054 (2006h:47088)

[4] K. Goebel and W. A. Kirk, Topics in Metric Fixed Point Theory, Cambridge Univ. Press, Cambridge, 1990. MR1074005 (92c:47070)

[5] W. A. Kirk, A fixed point theorem for mappings which do not increase distances, Amer. Math. Monthly 72 (1965), 1004-1006. MR0189009 (32:6436)

[6] W. A. Kirk and B. G. Kang, A fixed point theorem revisited, J. Korean Math. Soc. 34 (2) (1997), 285-291. MR1455542 (98e:47092)

[7] W. A. Kirk and B. Sims, Handbook of Metric Fixed Point Theory (W. A. Kirk and B. Sims, editors), Kluwer Academic Publishers, 2001. MR.1904271 (2003b:47002)

Departamento de Análisis Matemático, Universidad de Sevilla, P.O. Box 1160, 41080Sevilla, Spain

E-mail address: espinola@us.es 